Cahiers de recherches médiévales

\title{
Jean Corbechon, un traducteur encyclopédiste au
} $\mathrm{XIV}^{\mathrm{e}}$ siècle

\section{Bernard Ribémont}

\section{(2) OpenEdition \\ 12 Journals}

Édition électronique

URL : https://journals.openedition.org/crm/932

DOI : $10.4000 / \mathrm{crm} .932$

ISSN : 1955-2424

Éditeur

Honoré Champion

Édition imprimée

Date de publication : 15 février 1999

ISSN : 1272-9752

Référence électronique

Bernard Ribémont, " Jean Corbechon, un traducteur encyclopédiste au XIVe siècle », Cahiers de recherches médiévales [En ligne], 6| 1999, mis en ligne le 11 janvier 2007, consulté le 15 décembre 2022. URL : http://journals.openedition.org/crm/932 ; DOI : https://doi.org/10.4000/crm.932

Ce document a été généré automatiquement le 15 décembre 2022.

Tous droits réservés 


\title{
Jean Corbechon, un traducteur encyclopédiste au XIV ${ }^{\mathrm{e}}$ siècle
}

\author{
Bernard Ribémont
}

1 Le De proprietatibus rerum de Barthélemi l'Anglais fut, avec l'œuvre de Vincent de Beauvais, l'une des encyclopédies les plus populaires du Moyen Âge. Elle connut rapidement un grand nombre de traductions, dont celle, en français, commandée au frère augustin Jean Corbechon par le roi Charles V, et achevée en 1372. Cette œuvre offre un exemple intéressant des problèmes posés par la traduction d'un texte à caractère scientifique dans une langue en pleine mutation et qui ne possède pas encore l'équivalent de beaucoup de termes techniques latins. Se pose aussi la question de l'intervention du traducteur face à une matière que, au sens propre, il doit vulgariser. Nous étudierons donc dans cet article quelques éléments permettant de mieux cerner la réflexion de Corbechon devant son propre travail.

2 C'est dans sa dédicace au roi que Jean Corbechon exprime ses idées sur le travail qui lui a été confié ${ }^{1}$. La majeure partie de son prologue développe des arguments qui, comme S. Lusignan l'a bien montré, sont traditionnels chez les traducteurs agissant sous les ordres de Charles V. Le traducteur rappelle que l'idéal d'un roi est celui de "sapience » et, à l'appui de sa démonstration, on retrouve l'argumentation de la translatio studii, dont le mérite essentiel revient ici à Charlemagne, tête de pont d'une liste de puissants antiques parmi lesquels Alexandre et Jules César. La plus grande part de ce prologue entre donc dans une catégorie que l'on peut considérer comme topique au vu des autres traductions de l'époque, ainsi que le montre S. Lusignan :

3 La lecture de n'importe lequel des prologues dédicatoires de traduction montre d'emblée que le traducteur situe son œuvre dans une perspective de transmission ou d'appropriation du savoir au profit du pouvoir royal. Bien que ces prologues suivent rarement la topique de l'accessus ad auctorem des commentaires latins des auctoritates, aucun n'omet cependant de démontrer l'utilitas de l'œuvre pour son destinataire royal.

4 Dans la majorité des prologues, cette démonstration peut emprunter deux voies d'argumentation. Une première voie, qu'on pourrait qualifier d'éthique, démontre comment le contenu de l'œuvre traduite contribue à la formation morale ou à 
l'instruction politique du roi (...). Une seconde voie d'argumentation, de nature historique cette fois, illustre que les grands rois du passé qui ont marqué leur époque ont tous eu le souci de se procurer des livres et d'étudier. ${ }^{2}$

Une originalité de Jean Corbechon réside toutefois dans son appréciation du contenu encyclopédique du livre qu'il traduit. Le roi, trop occupé par ses fonctions, ne peut pas lire tous les ouvrages savants. Pourtant, à cause de son désir de connaissance et aussi à cause de sa fonction royale, il doit avoir une appréciation sur tout. Ce problème délicat peut être résolu au moyen de l'encyclopédie. On est proche ici des idées de Brunetto Latini dans le Livre dou Tresor, mais plus clairement exprimées et davantage soulignées. Le travail de Corbechon est pris dans une fonctionnalité qui va très au delà de considérations morales telles qu'on les rencontre au XIII ${ }^{\mathrm{e}}$ siècle. Il y a chez lui une appréciation très moderne du texte encyclopédique, accessible par la langue, devant servir à l'instruction du roi, mais aussi «au prouffit du peuple que Dieu vous a mis a gouverner », et permettant d'embrasser la somme des connaissances par abrégé, «pour ce que la vie d'un homme ne souffiroit mie pour lire les livres que vostre noble desir a assemblez».

On ne trouvera par contre que très peu concernant l'opération de traduction et la question de la langue. Corbechon se contente d'indiquer qu'il «translate le livre devant dit de latin en françoiz, le plus clerement que je pourroy ». Toutefois, il exprime parfois quelques remarques au sujet de sa traduction, en général pour en souligner la difficulté, comme par exemple dans le livre VI, à propos de l'enfance :

L'aage de l'enfant se fine au .vii ${ }^{\text {. }}$ an et la se commence le secont aage, que nous appellons enfance en françoiz, maiz en latin on l'appelle puericia, et en ce appert il que il y a plus grant deffaulte de langage en françoiz que en latin car il y a .vii. aages nommés par divers noms, desquelz il n'en y a que troiz en françoiz, c'est assavoir enfance, jeunesce et viellesce, et par ce on puet penser quele paine ce est de translater latin en françois (BN fr. 22531, fol. 102).

7 Il faut donc chercher au fil du texte les éléments de sa méthode : Corbechon en effet, à la différence de certains autres traducteurs, n'annonce pas sa conception de la traduction. Jean de Vignay par exemple, indique qu'il va rendre la lettre latine en français; Jean d'Antioche, frappé comme la plupart par les différences entre les deux langues, en fournit une analyse dans sa traduction du De inventione. Le "clerement» de Corbechon demeure bien vague et laisse toute latitude entre le littéral et l'adaptation, entre le résumé, l'extension et le décalque.

8 Cette largeur de champ, Corbechon va en fait la parcourir dans son souci de rendre le texte de Barthélemi le plus clair possible. Dans l'ensemble, le traducteur va tenter de serrer au plus près le contenu de l'encyclopédie latine. Mais cela n'est guère toujours possible, si l'on considère les différences de tournures et de vocabulaire entre le latin et le français: ces difficultés imposent un cheminement qui passe par la traduction littérale - compréhensible ou non -, l'adaptation, le résumé, l'omission ${ }^{3}$.

9 L'un des premiers problèmes auquel Corbechon va se heurter est celui de l'étymologie. L'on sait combien la méthode isidorienne avait marqué l'encyclopédisme du XIII ${ }^{\mathrm{e}}$ siècle : Barthélemi l'Anglais en particulier utilise beaucoup d'étymologies. Du point de vue de la traduction, la question est a priori fort complexe, la méthode étymologisante reposant sur les caractères du signifiant latin et, dans le cas le moins rigoureux, sur des jeux de mots qui dépendent donc étroitement de la langue.

Traduction des étymologies latines 

est confronté à une étymologie que Barthélemi prend en général chez Isidore de Séville. Je vais donner une liste de cas, illustrés par quelques exemples ${ }^{4}$. Cette approche typologique ne peut, il est clair, se concevoir selon des catégories strictes : les exemples montreront que, dans bien des cas, il y a des configurations mixtes.

1) Décalque ; trois cas se présentent alors :

2 a) le fait de démarquer le latin fonctionne en français et l'on obtient une proposition qui fait sens et une explication étymologisante satisfaisante :

L'or est denommé de l'air, (...) pour ce qu'il reluist plus fort par la reverberacion de l'air. (X, fol. 226va)

Alabandine est une pierre qui est ainsi nommee pour la region d'Alabande ou elle croist. (X, fol. 229a)

Charboncle est une pierre trés precieuse qui est ainsi appellee pour ce qu'elle est ardant et rouge comme un charbon. (X, fol. 229vb)

Arain est ainsi appellé pour l'air qu'il fait resplendir. (X, fol. 231a)

Fer est nommé de ferir (...) car le fer par sa durté fiert et debrise tous metauls. (X, fol. 232b)

La crapoudine est appellee noset et est une pierre precieuse soubzblanche ou vaire couleur qui croist en la teste du crapaut. (X, fol. 235vb)

Les maschoires sont dictes de maschier (A, fol. 69v).

Les dois sont ainsi appellez pour ce qu'ilz sont .x. (A, fol. 79) .
Aurum ab aura est dictum (...) eo quod repercusso aere plus refulget (fol. 151v).

Alanbandina est gemma, dicta ab Alabanda regione Asie (fol. 153).

Carbunculus lapis est preciosissimus, sic dictus, quia ut carbo est ignitus (fol. 154).

Es a splendore aeris est vocatum (fol. 155).

Ferrum a feriendo est dictum (...) sua enim duricie ferit (fol. 156).

Noset vel crapodine lapis est preciosus, subalbibus aut varius, qui in buffonis capite a quo extrahitur (fol. 158).

Mandibule a maducando sunt dicte (fol. 32). Digiti nuncupati sunt vel quia decem sunt... (fol. 37v)

b) La phrase fait sens mais Corbechon doit faire appel au grec ou au latin pour que l'étymologie soit compréhensible de son lecteur; au pire, il donne le mot latin et traduit son étymologie, qui doit souvent être obscure pour le non-latiniste ; parfois, il sent la nécessité d'ajouter une glose explicative (comme dans le cas de l'Arabie heureuse par exemple): 
La queux est une pierre qui est ainsi appellee pour ce que on y aguise le fer pour mieulx tranchier, car queu en grec c'est copper en latin. (X, fol. 229va)

Les pierres precieuses qui reluisent sont appellees gemmes en latin pour ce qu'elles sont cleres et reluisent comme la gome qui ist des arbres. (X, fol. 233a)

Oniche est une pierre d'Inde et d'Arabie qui a en soy couleur mellee aussi comme a l'ongle d'une personne; et pour ce est elle appellee oniche en grec qui est a dire ongle en latin. (X, fol. 235vb)

Aprés enfance, vient le secont aage, qui en françoiz n'a point de nom differenz du premier, maiz en latin on l'appelle puericia, et est ainsi appellé pour ce qu'en cel aage, il est encore aussi pur comme la prunelle de l'ueil (A, fol. 100)

Sapin est en latin appellé abies et est un arbre ainsi nonmé pour ce que en croissent il va plus hault que nul autre arbre (X, fol. 276v). ${ }^{5}$

...homme par abusion si est dit et denommé de terre, car il est composé non pas seulement de corps, qui est de terre, maiz aussi de l'ame qui est de nature esperituelle. Et de ce vient que en grec, homme est appellé, qui en latin est a dire halt eslevé, pour ce que par le gouvernement de l'ame il est eslevé de ça bas la hault, a la contemplacion de son createur (A, fol. 33v).

En Arrabie, a moult d'arbres qui portent encens, et moult d'aultres biens odorans pour lesquelz les Grieux appellent celle region Eudemon, qui est a dire en françoys Bonne-a-Dieu et les Latins l'appellent region benoitte (A, fol. 228).
Cos est lapis sic dictus, eo quod ferrum ad incidendum acuat. cotis enim greco sermone incisio dicitur (fol. 154).

Gemma dicitur eo quod luceat instar gummi (fol. 156v).

Onichum lapis est indicus et arabicus, permixtum in se habet colorem ad similitudinem humani unguis (...). Greci enim unguem onicem dicunt (fol. 158v).

Cui succedit pueritia (...) dicta a pubertate vel a pupilla (...), quia adhuc pueri sunt puri ut pupilla (fol. 50v).

Abies est arboris, sic dicte ab eundo, eo quod pre ceteris arboribus longe eat (fol. 167).

Abusive est homo ab humo dictus, cum ex societate non tantum corporis sed anime sit concretus. Et ideo grece antropos id est sursum erectus appellatur, eo quod ab humo ad contemplationem suis artificis spiritus regimine sublevatur (fol. 10v).

Ibi enim maxime abundant arbores thurifere et alie odorifere propter quam sacram aromatum fragantiam, Greci eam Endemon, Latini vero beatam, vocaverunt (fol. 137).

c) Corbechon traduit l'étymologie mais le lecteur non latiniste et surtout non familier d'Isidore ne voit guère le rapport entre le mot français et son explication étymologique.

La pierre est aussi ainsi appellee pour ce qu'elle perce le pié quant on la foule trop fort. (X, fol. 236a)

La poudre est ainsi appellee pour ce qu'elle est boutee et hurtee du vent. (X, fol. 237b)

...pour ce est il appellé souffre selon Ysidore, qui est a dire seul ardant. (X, fol. 239b)

Le rosel est ainsi appellé pour ce que il croist tantost (X, fol. 277v.)

Arbre (...) est ainsi appellé pour les champs et la terre a qui il se tient en fichant ces racines dedens (X,fol. 273v). ${ }^{6}$
Petra a penetrando est dicta, penetrat enim pedem (fol. 158v).

Pulvis dicitur eo quod a vento pulsetur (fol. 159v).

...et ideo vocatur sulphur secundum Isidorum quasi solum urens (fol. 161).

Arundo est dicta eo quod cito arescat (fol. 167v).

Arbor (...) ab arvis nomen sumpsit (fol. 162v). 
Ache est une herbe commune qui est congneüe de toutes gens et est appellee hache, si comme dit Ysidoire au .xvii ${ }^{\mathrm{e}}$. livre, pour ce que les anciens la mettoient sur le chief de ceulx qui avoient victoire en bataille (X, fol. 279).

Avoingne (...) est appellee avoinne pour ce qu'elle vient tost aprés ce qu'elle est senmee (X, fol. 279v). Ayl est ainsi appellé pour ce qu['il] put (X, fol. 278). ...est appellee Cappua pour ce que son territoire comprent tous blefs et fruis qui sont neccessaire a vie humaine (A, fol. 234v).
Apium est communis herba, omnibus fere nota, ex eo sic vocata (...) quod apicis id est capitis triumphantium quondam fuit ornamentum (fol. 168v).

...est sic dicta, eo quod postquam seminatur, cito adveniat... (fol 169).

Alium ab olendo est dictum, eo quod olat (fol. 168).

A capacitate dicta sic, eo quod ejus terra omnem vite fructum capiat (fol. 141). l'étymologie pour que les mots français se correspondent, au moins de manière consonnante; par exemple :

Serpent est ainsi appellé pour ce qu'il serpe $(\mathrm{X}$, fol. 319)
Serpens est dictus, eo quod occultis serpit (fol. 234v).

Au pire, la phrase française obtenue n'a aucun sens :

Le pallis est fait de paulx aguisés es deux bous qui en latin sont appellés sudes (...) pour ce que ilz cousent les parties de la haye l'une a l'autre (X, fol. $314 \mathrm{v})$.
Sudes dicitur a sudo (fol. 195v).

2) Création: Corbechon invente des étymologies en jouant le plus souvent sur la consonnance; comme par exemple l'étymologie du mot «France » d'après «Francion » ou le boucher $«$ Francus $»^{7}$. Deux cas peuvent se produire :

a) jeu sur le français :

Les potages sont ainsi appellés pour ce que on les cuit en pot $(\mathrm{X}$, fol. 298).

La balance est ainsi appellee pour ce que elle bale de legier (X, fol. 341).

Le chauchetrappe donc est une herbe poingnant qui navre et pique les piés de ceulx qui passent et les fait saillir par dessus et tripper et pour ce est elle appellee en France chauchetreppe (X, fol. 307v).

b) jeu sur latin et français :
Legumina ab eligendo sunt dicta quasi electa (fol. 183).

Statera a stando est dicta (fol. 263v).

Paliurus nam est herba aculeata, pedes vulnerans transeuntis (fol. 192). 
Chardon est en latin appellé tribulus (...) pour ce qui donne paine et tribulacion aux mains qui le touchent (...). Et pour tant par y bien que le chardon est a bon droit appellé tribulus en latin, car il tribole tout ce qui approuche de lui (X, fol. 317).

Pas d'équivalent chez Barthélemi.

c) jeu sur le latin: Corbechon prend une propriété physique donnée par Barthélemi et la transforme en étymologie :

Ceste pierre (ematite) selon le Plateaire est de froide et seiche complexion et a vertu de restraindre le flux de sanc selon l'interpretacion de son nom. (X, fol. 231vb)
Dicitur ab emath quod est sanguis et thites quod est sisto (fol. 155v).

21 3) Élimination: Corbechon omet l'étymologie ou la retranscrit en explication de type naturaliste - c'est-à-dire comme caractéristique physique de l'objet étudié-, sans qu'apparaisse la dimension lexicographique.

Leton (...) est un dur metal: par dessus il reluist aussi comme or. (X, fol. 227b)

Omission de Corbechon.

La perle est engendree de la rousee du ciel que les poissons ou elle croist reçoivent en certain temps de l'an et n'en treuve l'en oncques que un en un lieu. (X, fol. 234vb)

Arrabie est une province sacree pour l'encens qui y croist (A, fol. 228).

Palme est un arbre de vittoire qui est ains appellé pour ce que jadis on la metoit en la paume de ceulx qui avoient vittoire (X, fol. 304v).
Aurichalcum (...) est dictum eo quod cum fit aes sive cuprum resplendet superficialiter sicut aurum, aes enim graeco sermone chalcum vocatur (fol. 152).

Asbestos (...) ab igne nomen sortitus (fol. 153).

ex quibus margaritis quedam uniones nuncupantur habentes aptum nomen, eo quod tantum unum et nunquam duo vel plures reperiuntur (fol. 157v).

Arabia Asye est provincia sacra id est thurifera nuncupata (fol. 137).

Palma est arbor victorialis sic dicta (...) quod caput victoris sive optinentis victoriam est ornatum, vel quia adpenses ad modum palme hominis habent ramos (fol. 19).

Dans les cas d'omission, il est difficile de se faire une opinion précise sur la réflexion de Corbechon. Certaines étymologies de l'original latin ne seraient en effet guère difficiles à traduire, comme celle-ci: Mesopotamia greciam ethimologiam possidet eo quod duobus fluviis ambiatur. Il arrive, lorsque Barthélemi donne plusieurs étymologies, que Corbechon n'en retienne qu'une seule, comme dans le cas d'aries. On peut noter qu'il en élimine un certain nombre d'absurdes, comme celle d'equus : equus a multis etiam sonipes nominatur eo quod pedibus sonet ou celle du déjeuner : potius dicitur (cens) a scinos quod est canis propter carentiam et defectum caritatis; unusquisque enim suam cenem presumit ad manducandum. Mais, dans la mesure où il en reproduit aussi bon nombre de douteuses, il est bien difficile de trancher en la matière.

4) Rétablissement: quelquefois, rarement il est vrai, Corbechon rétablit une étymologie incomplète ou incompréhensible de Barthélemi en retournant à la source d'Isidore ou de Papias. Ce quatrième cas, de manière encore plus cruciale que les autres, 
n'offre toutefois aucune certitude dans la méconnaissance où nous sommes des manuscrits du De proprietatibus rerum utilisés par Corbechon.

\begin{tabular}{|l|l|l|l|}
\hline $\begin{array}{l}\text { La mort est ainsy } \\
\text { appellee pour ce qu'elle } \\
\text { mort amerement (X, } \\
\text { fol. 74v). }\end{array}$ & $\begin{array}{l}\text { Mors dicta est eo quod sit } \\
\text { amara vel a Marte qui } \\
\text { mortem fingitur efficere } \\
\text { (fol.50v). }\end{array}$ & $\begin{array}{l}\text { Mors dicta quod sit amara vel a Marte qui est } \\
\text { effector mortium sive mors a morsu hominis } \\
\text { primi, quod vetitae arboris pomum mordens } \\
\text { mortem incurrit (Etym., 11, 2, 31). }\end{array}$ \\
\hline
\end{tabular}

...et est appellee Gaule la Brayee pour ce que ceulz du pays souloient porter longues brayes (A, fol. 246).
Pas d'équivalent chez Barthélemi qui cite la Gallia Bracata (fol. 143v) à propos de la Gaule Narbonnaise.

Cette série d'exemples, en forme de sondage, montre bien que Jean Corbechon est un véritable «translateur » qui, s'il n'explicite pas son rapport au procès de traduction, n'en travaille pas moins sur le texte de Barthélemi, au niveau de la langue comme du contenu; même si les cas ne forment pas la majorité, il est notable en particulier de considérer des exemples où l'on voit le traducteur corriger son modèle pour revenir à une étymologie qu'il juge plus précise, rétablissant, entre autres, la lettre d'Isidore ou de Papias, voire de Pline. A contrario, on trouve aussi des exemples où le traducteur est pris en défaut de mauvaise compréhension, d'oubli : même si, globalement, Corbechon apparaît comme un bon traducteur, il existe des failles dans son système. Celles-ci recouvrent cependant un ensemble assez faible de cas qui ne saurait occulter la ferme volonté de Corbechon de rendre clair le texte de Barthélemi.

parcours de l'ensemble du Livre des proprietés des choses confirme cet engagement du traducteur au devant du texte latin: fidèle lorsqu'il le peut et lorsque le discours lui paraît lui-même clair, résumant des passages jugés trop long, glosant des termes obscurs ou des propositions peu explicites, ajoutant de-ci de-là quelques propos personnels, rétablissant parfois certaines sources, Corbechon ne joue que très peu le jeu de la facilité : il préfère celui du travail sur le texte.

Le travail du texte

2 La première chose qui frappe à la lecture globale de la traduction de Corbechon est la qualité littéraire de celle-ci. Corbechon fait visiblement des efforts de ce point de vue, condensant lorsque son prédécesseur est trop prolixe, ajoutant des explications, utilisant des comparaisons; il s'efforce en outre de maintenir - surtout dans les livres plus théoriques comme ceux réservés aux substances séparées ou à des théories sur les humeurs, les éléments, etc. - une logique démonstrative marquée par les "car", "donc», «sicomme il appert », «cy a entendre », «comme ainsi soit que », etc. Il traduit ainsi ce que Barthélemi exprime avec force igitur, enim, unde, eo quod, etc. Il reproduit en général la juxtaposition barthélemienne des propriétés d'un même objet, que le latin rend par item, en traduisant par « derechief». Voici par exemple le cas de la chaleur, où l'on peut voir ce type d'articulation:

Chaleur donc est une qualité qui appartient aux elemens, et par especial a l'element $\mathrm{du}$ feu. Et ceste qualité est active souverainement, sicomme il appert en aucunes euvres, car nulles des aultres qualitez ne euvrent si tost, ne si prestement comme fait la chaleur. Derechief, la chaleur est cause principale de la generation de toutes choses qui prennent leur estre par generation. $\underline{\mathrm{Cy}}$ (a) a entendre que ilz sont deux 
manieres de chaleur; l'une si est la chaleur souleil et du ciel, et est cause de generation et de conservation, sicomme il appert aucunes foiz es nues, esquelles sont engendrees les raynes par la vertu de la vertu du souleil, et cetera. L'autre chaleur est des elemens, et ceste cy si est aucunes foiz cause de corrupcion, sicomme il appert ou mirouer ardant, qui art le drap pour cause de la chaleur qui vient des rays de l'air qui sont brisez dessus le mirouer.

Derechief, la chaleur si ramaine les choses de ça bas avecques celles de lassuz, sicomme il appert quant la chaleur monte de bas en hault, elle convertist la terre en eaue, et l'eaue en air, et l'air en feu, par sa vertu. Derechief, la chaleur si amollie aucunes foiz les choses dures, sicomme il appert en la cire et es metaulz qui par chaleur se fondent et sont amolliez. Derechief, la chaleur si endurcist aucunes foiz les choses molles, sicomme il appert de un euf qui est cler et mol de sa nature, qui est endormi par la chaleur du feu (A, fol. 47v-48).

En général, Corbechon comprend le plus souvent la logique du texte qu'il a sous les yeux et il la rend avec une habileté certaine. Du point de vue de la structure du texte, Corbechon entend elle aussi la respecter; mais, dans le détail de sa traduction, on peut enregistrer quelques écarts avec le De proprietatibus rerum, ce qui conduit Corbechon à quelques attitudes contradictoires.

D'une part en effet, Corbechon manifeste dans sa dédicace au roi une conscience de l'emploi qui peut être fait du texte encyclopédique. La structure déjà rigoureuse du $D e$ proprietatibus rerum fait de ce dernier un ouvrage consultable, dans lequel d'ailleurs l'usage de l'ordre alphabétique est très fréquent. Ce que Corbechon annonce dans son propre prologue va donc dans le même sens. Or, le désir littéraire du traducteur l'amène aussi parfois à produire un ensemble davantage conçu comme devant être lu de manière linéaire. Globalement, en traducteur qui se veut avant tout fidèle, Corbechon reprend la structure du De proprietatibus rerum; cependant, si le traducteur ne déroge guère à la conception barthélemienne de l'encyclopédie - à l'exception de quelques considérations politiques contemporaines -, quelques indices sont révélateurs d'une déviance vers un texte plus linéaire, au moins par parties.

Barthélemi traite souvent les chapitres des livres du De proprietatibus rerum comme des entités séparées, des rubriques consultables pour le lecteur; ceci ne l'empêche pas de ménager quelques transitions, en particulier lorsqu'il passe d'une grande unité à une autre: la fin d'un livre est en général marquée et sert également d'annonce du prochain $^{8}$. Tel est par exemple le cas du passage aux humeurs au livre IV (fin du chap.V). Si Corbechon reprend la structure de près, il ajoute cependant de temps à autre une phrase de transition de son propre fait, permettant, dans le cadre d'une lecture continue, de passer plus agréablement d'une rubrique à la suivante. Si Barthélemi ne clôt pas son prologue par exemple, Corbechon le fait: «Ci fault le prologue de l'aucteur ». Autre exemple: au livre III, pour passer du premier au second chapitre, le traducteur rajoute la transition suivante: «Ce est donc raison que notre consideracion, en traittant de l'omme, si preigne son commencement a l'ame, tant comme a la plus digne partie qui sont en homme» (A, fol. 33v).

Par ailleurs, Corbechon est souvent gêné par l'ordre alphabétique, outil par excellence de la consultation. Barthélemi, comme il l'avait annoncé dans son prologue, utilise ce système de classement dès qu'il s'agit d'objets physiques. L'ordre n'est toutefois pas rigoureux et Barthélemi ne suit pas le modèle de Papias, que pourtant il connait bien, et se contente de l'ordre limité à la première lettre. Corbechon semble pris entre son intention de fidélité au texte et une logique structurante du discours selon l'ordre 
alphabétique. Il choisit finalement, au détriment de l'aisance de la consultation, de suivre l'ordre latin, en précisant toutefois, chaque fois quasiment que le cas se présente, qu'il place tel élément parce que son nom latin le situe à cette place : «toute serpent qui se ploie et s'entortille est appellee anguis, et pour ce en sont cy mise les proprietés avec les bestes dont les noms commencent par .a.» (A, fol. 332v); «Il est trois manieres de tuyaux dont l'un est aromatique, l'autre est usual et l'autre est pour escripre. Et est chascune de ses trois en latin appelle calamus, et pour ce sont ilz cy mis entre les arbres et les herbes dont le noms se commencent par la lectre de .c.» (X, fol. 283), etc.

Le troisième élément de détail que l'on peut enregistrer à propos du travail littéraire de Jean Corbechon touche aux rappels qui se trouvent dans le texte. Il arrive en effet que Barthélemi indique à son lecteur qu'il pourra trouver des informations complémentaires concernant un sujet à un autre endroit de l'ouvrage : à propos de la chèvre par exemple, Barthélemi invite son lecteur à quere infra in lettera i. de irco (fol. 218); il fait de même pour le chameau: alias proprietates quere infra de dromaderio (fol. 216v). Corbechon ne donne pas ces renvois qu'il juge semble-t-il inutiles et préfère se ranger à une attitude de concision qui lui fait terminer beaucoup de chapitres par une indication quelque peu laconique du type «a tant souffise pour cette matiere».

Mais que l'on ne s'y trompe pas, en considérant que Corbechon «bâcle» en ce cas sa traduction: d'une part, Barthélemi lui-même termine très souvent ses rubriques de cette façon: lorsque Corbechon par exemple clôt ainsi le quatrième chapitre du livre IV, «ce qui est dit de l'air froit et chaut, par comparaison a la moisteur, si souffise a present ", Barthélemi avait conclu ainsi sed de aere frigido ad presens hec dicta sufficiant. D'autre part, il existe souvent un réel désir de concision de Corbechon par rapport au latin, qu'en maintes occasions il résume: tel est le cas par exemple pour la pierre appellée enidros au livre XVI, cas au demeurant intéressant dans lequel Barthélemi critiquait sa source marbodienne :

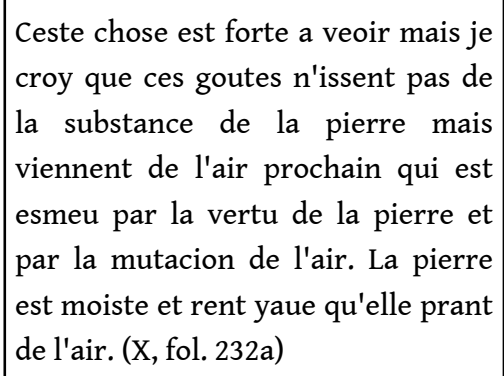

Et dicitur ibidem quod difficile est hujusmodi reddere rationem, quia si gutta defluit de lapidis substantia, quare non minor efficitur, vel omnino liquescit, si vero res vel aliud ipsum ingreditur, quia ingrediens non repellit ingredientem? Potest tamen esse, ut mihi videtur, quod virtus lapidis condensat in aquam aerem sibi vicinus propinquantem, et jam videatur exire de substantia lapidis, quod ingreditur de substantia aeris circumstantis (fol. 156).

Cette attitude est consciente chez Corbechon, car il l'explique lui-même quelquefois, jugeant que Barthélemi a laissé quelques redondances dans son texte. Au livre XVII par exemple, il ne suit pas son prédécesseur et condense, en l'expliquant, les deux chapitres De stacte et De storace ${ }^{9}$. Il termine ainsi le chapitre sur le storax : «Il est une autre goute que on appelle states qui yst de la mierre qui est une mesme chose, state et la mierree de quoy nous avons parlé cy dessus, et pour ce nous en passons a tant sans en faire chappitre especial» $(\mathrm{A}$, fol. 313v). Il révèle une démarche analogue avec le calamus. Barthélemi en effet écrit trois chapitres sur ce végétal, correspondant aux trois variétés: calamus aromaticus, calamus usualis, calamus scripturalis. Dans le dernier 
chapitre, Barthélemi reprend les affirmations de Pline sur l'arundo: Corbechon ne traduit pas ce passage, et se contente de renvoyer à Pline. Comme l'a remarqué G. Sodigné-Costes dans le cas du livre XVII, Corbechon se livre aussi, dans le cadre de la réduction, à de véritables restructurations du texte barthélemien; tel est le cas du chapitre sur le froment, comme dans celui sur les arbres aromatiques. Dans ce dernier cas, Barthélemi travaille à partir de l'ouvrage pseudo-aristotélicien, le De plantis, auquel il rajoute de nombreux commentaires. La comparaison de la traduction de Corbechon, du De plantis et du De proprietatibus rerum, montre que Corbechon est probablement revenu à la source pour retraduire le texte, omettant les commentaires de son prédécesseur ${ }^{10}$. On peut multiplier les exemples au fil du texte qui montre chez Corbechon une volonté de réduire le texte barthélemien. On y verra sans doute un souci pédagogique émanant du vulgarisateur qui veut aller à l'essentiel.

On peut à ce propos s'interroger sur les parties les plus complexes, d'un point de vue scientifique, du De proprietatibus rerum. On peut remarquer en effet que Corbechon résume, parfois de façon drastique, les passages qui lui paraissent techniquement complexes. Ce n'est pas le lieu de s'interroger ici - ce qui d'ailleurs est un faux problème - sur le degré de compréhension de Corbechon lui-même : si l'on veut fournir une vision négative du traducteur, on peut considérer qu'il omet ce qu'il ne comprend pas. On peut considérer aussi que sa conception de l'encyclopédie en vernaculaire est un peu différente de celle de Barthélemi pour l'encyclopédie latine. Ce qui importe est le résultat obtenu et le fruit qui est effectivement livré au public, fruit qui, dans sa forme, montre effectivement le souci de simplifier un certain nombre de questions et donc peut-être de s'adresser à un plus vaste horizon de lecture. Dans le cas de certains développements barthélemiens sur l'optique par exemple, Corbechon a effectivement tendance à «passer à la ligne». Voici par exemple ce que décrit Barthélemi au livre III, figure à l'appui ${ }^{11}$, pour donner, à la suite d'Aristote, la seconde des conditions nécessaires à la visibilité d'un objet :
Secundum est oppositio visibilis contra visum quia visus tali modo videndi non videt, nisis rem illam cujus partibus venit species super lineas rectas cadentes in centrum oculi, que omnes linee ducte a singulis partibus rei faciunt unam piramidem cujus conus est in pupilla et basis in re visa, ut patet in hac figura.
Axis visualis est radius ille sive linea que intellegitur duci a medio visus ad punctum rei vise directe ad oppositionem in medio visus vel oculi, ut patet in hac figura : a. enim et .b. sunt axes quando videns directe intuetur punctus .c. Quando vero intuetur punctus .d., tunc ille due .b. .c. line exeuntes $a b$ oculis dicuntur axes quia exeunt in medium .ab. oculi medio ad rem visam (fol. 14).

Corbechon donne alors la «traduction » suivante : « la seconde si est que la chose visible soit a l'apposite de la veue » $(\mathrm{X}$, fol. 23v). Il abrège considérablement les explications de Barthélemi sur les éclipses au livre VIII (latin, fol.189v, X, fol.131). Dans la partie mathématique (livre XIX) du De proprietatibus rerum, Corbechon omet entièrement le chapitre sur les nombres plans et solides (fol. 259-259v). Il est clair - on pourrait multiplier les exemples - que beaucoup de chapitres techniques du De proprietatibus rerum sont abrégés par Corbechon. Il ne faut cependant pas trop rapidement conclure à l'indigence scientifique de Corbechon, même si l'on peut penser à quelques faiblesses de sa part, ou, pour le moins, un manque d'intérêt pour certaines digressions trop abstraites: sa traduction sur les différentes divisions des nombres entiers par exemple est très fidèle, très claire et même, il rajoute la référence à Boèce que Barthélemi ne 
donne pas ${ }^{12}$. Il s'agit donc avant tout pour Corbechon de simplifier, en fonction du public auquel il s'adresse.

De même que parfois, comme nous l'avons vu, Corbechon rétablit certaines étymologies, il lui arrive aussi de corriger les sources citées par Barthélemi ou bien de rétablir des références omises dans le latin. Dans le livre III, au chapitre XIII, Barthélemi attribue à Isidore l'opinion selon laquelle l'âme est immortelle car, dans le cas contraire, elle ne pourrait être à l'image de Dieu; Corbechon rectifie en citant la vraie source, à savoir Cassiodore ${ }^{13}$. Il lui arrive également de rétablir des références qu'il juge trop vagues: Barthélemi emploie souvent le terme de Commentator qui, au XIII ${ }^{e}$ siècle, désigne généralement Averroès ; chez notre encyclopédiste cependant, il désigne assez souvent d'autres autorités du commentaire, comme Alfred de Sareshel. Corbechon va quelquefois plus loin en vérifiant la source, afin de préciser de quel commentateur il s'agit. Au livre IX, un ut dicit Commentator (fol. 96) de Barthélemi devient chez Corbechon «sicomme dit Gallien» (X, fol. 145); le traducteur a d'ailleurs raison car la source est un recueil de sentences galéniques regroupées sous le titre d'Hippocratis Aphorismi. Au livre XIX, à propos de la couleur albus, Barthélemi cite le Commentator (fol. 240) que Corbechon rétablit bien en Averroès (X, fol. 324v); en ce même lieu, là où Barthélemi cite un lib. .xix. d'Aristote, Corbechon précise "ou livre .xix. des bestes ». De même, le frère augustin connaît bien les œuvres de l'évêque d'Hippone et ne manque pas, par endroits, de rajouter quelques références augustiniennes qui ne figurent pas dans le latin; comme au même chapitre du livre III par exemple : «Et pour tant elle ne se puet reposer fors en celui qui est trés bon, car son lieu si est Dieu, a qui elle tent par amour et par desir, et c'est ce que dit celui meismes saint Augustin, ou livre de ses Confessions: «Sire tu nous as faiz pour toy, et notre cuer n'est oncques en paix jusques a tant que il se repose en toy» ( $\mathrm{A}$, fol. 38v). C'est ainsi que Corbechon se plaît quelquefois à remonter jusqu'à la Bible, là où Barthélemi ne citait que d'après Isidore ; au livre XV par exemple, à propos de la malédiction de Cham, Barthélemi explicite sa source isidorienne et en reste là (A, fol. 141); Corbechon rappelle, après avoir lui aussi cité Isidore, « sicomme il appert en la Bible » (A, fol. 234v).

Dans le même ordre d'idée, il arrive à Corbechon de compléter des références de Barthélemi: au livre IX par exemple, Barthélemi fait référence, à propos des cauchemars, à l'Évangile de Matthieu $(27,11)$. Corbechon précise davantage en donnant des indications, non fournies par Barthélemi (fol.99v), sur l'histoire dont il est question: «et plus de fantasies aviennent a ceuls qui dorment par nuit que a ceuls qui veillent par jour, sicomme il appert de la fame Pilate qui en dormant par nuit ot moult a souffrir en sa fantasie pour Jhesus Crist » $\mathrm{X}$, fol. 150). Cette démarche de complément par rapport à la sainte Écriture est assez fréquente chez le traducteur, tout particulièrement dans le livre VI, où Corbechon aime à raconter de courts extraits de la Bible. Il raconte ainsi, là où Barthélemi ne fait qu'une référence lapidaire à la source, l'histoire de Ruben, fils aîné de Jacob, celle de Balthazar, celle d'Élisée ${ }^{14}$.

Enfin, on pourra remarquer le désir de Corbechon d'agrémenter son texte de quelques brefs commentaires sur le nom des auctores ou sur une citation. Il n'est pas rare en effet que le traducteur précise, au moyen d'une courte remarque, une «caractéristique» de l'auteur cité : on trouve ainsi «un ancien maitre qui fut appellé Erodoque» (A, fol. 247v) ; «Josephus, ce grant maitre des Juifs» (X, fol. ) ou le «grant docteur des Juifs» (X, fol. 311). 
(c) un certain nombre de références présentes dans le latin. Il est un cas semble-t-il où cette démarche, presque systématique, révèle un parti-pris de Corbechon. On peut en effet noter que ce dernier est particulièrement réticent à l'égard des poètes de l'Antiquité. Presque à chaque fois que Barthélemi cite un poète de mémoire, citation assortie d'un unde poeta, Corbechon omet et l'allusion au poète anonyme, et la citation. Le livre XVII du De proprietatibus rerum par exemple, consacré aux végétaux, contient un certain nombre de références aux Géorgiques et de citations de Virgile ; Corbechon les omet quasiment toutes ${ }^{15}$. S'il garde la référence au nom d'un poète, Horace et Lucain en particulier, il omet la citation lorsqu'elle figure dans le latin; Pétrone, cité par Barthélemi à propos des œufs de corbeaux (lib. XIX, cap. 84), est omis par Corbechon. On peut donc penser que notre frère de l'Ordre des Augustins n'ait guère apprécié les poètes latins du point de vue de l'encyclopédisme. De la même façon que l'on peut noter un certain nombre de réticences, de Barthélemi lui-même, pour la mythologie, on trouve chez le vulgarisateur français un état de méfiance très net pour l'Antiquité païenne non scientifique. Peut-être Corbechon jugeait-il que la «fiction des pouettes » pouvait présenter quelque danger pour le lecteur? Plus probablement - Corbechon étant un lettré - il faut mettre cette attitude au rang d'un choix à caractère "positif» d'une part, religieux de l'autre, qui vise à sélectionner ce qui explique effectivement les propriétés des choses de la nature et qui peut aussi, à l'occasion, servir à l'édification du lecteur.

41 Les quelques exemples que nous venons de donner, et que l'on peut multiplier au fil des livres du De proprietatibus rerum, montrent bien qu'il existe une dynamique de la traduction, dans le sens que Corbechon n'hésite pas à intervenir sur le texte de Barthélemi dans une direction qui lui paraît au mieux orienter la lecture du texte vers une meilleure compréhension et une meilleure saisie des données exposées. "Translateur", Corbechon l'est donc à double titre : dans le fait que, tout en demeurant globalement assez fidèle au texte, il ne se laisse aller que très peu souvent à la littéralité; en second lieu, par une attitude qui révèle en lui le vulgarisateur, dans un sens plus moderne. Il semble qu'il y ait un décalage, certes limité, mais réel, entre le De proprietatibus rerum et le Livre des proprietés des choses, le second s'adressant davantage à un public plus large, pour lequel Corbechon se transforme parfois en commentateur; ce décalage, qui existe a priori par le fait de langue, se marque donc aussi par certains contenus.

42 C'est dans les interventions directes de l'auteur que l'on peut mesurer une telle attitude qui montre à la fois l'engagement de Jean Corbechon par rapport à son texte et, également, son désir d'être plus clair, de mettre son propos à la portée de ceux qui n'ont pas en mémoire les traités d'Aristote et de ses commentateurs.

On peut tenter de classer les interventions directes de Corbechon en trois catégories :

1) remarques concernant la langue ou la traduction. Nous avons déjà rencontré chez Corbechon certains propos justifiant ou expliquant sa façon de traduire ou d'organiser son texte: en particulier, rappelons-le, il note la différence entre le terme français et le mot latin lorsque l'ordre alphabétique est perturbé. Il lui arrive également de rappeler 
que tel ou tel terme n'a pas de correspondant en français. Nous avons vu le cas des âges de l'homme. Corbechon fait de même pour les frumenta, au livre XVII : «tout grain de quoy on fait pain et qui croist en un espi est appellé fourment, si comme segle et orge et leurs semblables, si comme dit Ysidoire et Plinius, mais ceste maniere de parler n'est point en usaige en notre langaige, car tous telz grains nous appellons blé mais non pas froument» (A, fol. 291). Il note également que «sauls est en France appellé osier » et que la lappe, «en France, on l'appelle la fueille aux tigneux». On peut multiplier les exemples de ce type. Ces remarques liées à la langue française pourraient être considérées comme des interventions de l'auteur mettant en jeu son sens de la réalité, de l'observation. Un exemple révèle bien cette fragilité des frontières; c'est celui de l'« herbe sauvage » qui traduit le latin gramen : «l'erbe sauvage est aucune fois appellee a Paris herbe vert que on pouldre par les maisons et semble estre blef mais non est » (A, fol.293). Un exemple métrologique révèle, dans un sens identique, la volonté que Corbechon a de bien faire comprendre son texte au lecteur français : il convertit par exemple, au livre XVIII - où Barthélemi parle de la distance en mille parcourue par un dromadaire en un jour - les mille en lieues françaises (A, fol.347v). On peut toutefois considérer une classe à part, qui fera l'objet de notre seconde catégorie, dans laquelle Corbechon rend compte, non plus de faits de langue contemporains, mais de scènes, d'expériences auxquelles il dit avoir assisté.

2) remarques personnelles liées à l'observation: dans un ouvrage basé sur la compilation et dans lequel le poids de l'autorité est fondamental, il ne faut pas s'attendre à trouver beaucoup de comptes rendus d'expériences. Barthélemi l'Anglais écrit un livre à partir des livres et ne prétend pas faire partager à son lecteur une chronique de l'avancée scientifique de son temps. En tant que traducteur et en tant que clerc, Corbechon est doublement aligné sur un tel état d'esprit, à une époque où, en dépit de quelques premières avancées, on est encore fort loin de la science expérimentale; par sa nature en outre, l'encyclopédisme médiéval est particulièrement éloigné de tout mouvement pouvant conduire à une telle science. Toutefois, Corbechon agrémente quelquefois sa traduction de quelques remarques personnelles qui soulignent le texte de l'autorité de la vérification expérimentale. Tel est le cas par exemple d'une propriété médicinale de l'héliotrope: «l'eaue et le jus de ceste herbe vault moult contre la frenasie quant on met sus les temples et sus le front du malade et ce ay je veu l'esperiance a Paris en ma presence» (X, fol. 288v). Il note également la présence de la choucroute dans les régions de l'Est: «le cabus est bon en viande et en medicine et par especial en Lorenne et en Alemaigne ou on en fait une composte qui dure moult longuement et la mengüet on en quaresme en vin aigre et a la mostarde " (A, fol.298). Au chapitre sur la salive du livre V, Corbechon rajoute à sa traduction la remarque suivante (souligné par nous) :

Derechief, la salive de homme jeun si a une couverte vertu corrompant; car se un homme est navré de nouvel et on met de la salive d'un homme jeun dedens la playe, elle blece et corrompt le sanc; et c'est la cause, sicomme je croy, pourquoy aucuns archiers et arbalestiers moillent le fer de leurs flesches de leurs salives car elles en sont plus nuisans au corps de leur adversaire (A, fol. 73v).

A propos de la Grande Ourse (ici «Arthurus»), il ajoute également un court propos à caractère " populaire » : « ce signe est appellé du peuple le char saint Martin, car il y a .iiii. estelles l'une contre l'autre comme iiii. roues et trois devant comme les chevaulx » (A, fol. 164). Un chapitre du livre IX sur le temps est occasion à quelques remarques d'ordre contemporain : 
Oultre l'an usual et commun, il est un autre an qui est selon la coustume ou la neccessité du païs, sicomme es lettres du pape l'an commence à Noel, et es lettres $\mathrm{du}$ roy de France, il commance a l'Anonciacion Notre Dame, et ainsi est il es escriptures ou on trouve diverses assignacions de l'an (X, fol. 144v).

Ces quelques exemples, au fil du Livre des proprietés des choses, montrent en Corbechon un traducteur qui n'hésite donc pas à insérer son propre «je» dans le texte, le plus souvent pour ajouter quelque précision touchant à l'ordre contemporain des choses, afin de rendre son texte le plus lisible possible, c'est-à-dire le plus compréhensible, le plus «parlant» au niveau de l'illustration par l'exemple et, en dernier lieu, le plus agréable qu'il est permis. L'intervention de ce «je » va se faire également, dans un cadre d'autorité, lorsque Corbechon est conduit à réfléchir sur les propos compilés par Barthélemi ; tel est l'objet de notre troisième catégorie d'intervention du traducteur.

3) prise de position devant un choix provoqué par la percussion de deux sources ou, ce qui est voisin, manifestation d'une critique vis à vis d'une opinion retransmise ou formulée par Barthélemi :

$\mathrm{Au}$ livre XI, consacré à la sphère de l'air, Barthélemi donne deux opinions contradictoires concernant le tonnerre. La première est prêtée au Philosophus (Aristote en principe) et à Isidore: selon cette affirmation, le tonnerre serait le bruit provoqué par le vent contenu dans les nuages. La seconde opinion, secundum Aristotelem, indique que le tonnerre est le bruit d'une vapeur portée à haute température qui se refroidit brusquement dans un nuage aqueux. (fol. 109v). Corbechon, qui se trouve de fait face à deux opinions différentes émanant toutes deux d'Aristote, choisit la dernière et précise que :

Selon Aristote, ou livre de Metheores, le tonnoirre n'est autre chose que le feu qui est estaint en la nue, car les vappeurs qui sont chaudes et seiches qui sont traiites hault et enflambees par la chaleur du souleil, quant elles se boutent dedens une nue plaine d'eaue, elles sont tantost estaintes, et en estaignant ils font le son du tonnoire, aussi comme fait un feu chault quant on le boute en l'eaue. Ceste oppinion du tonnoirre est la vraye entencion de Aristote et tout ce qu'il en dit autrement c'est en recusant les oppinions des autres (A, fol. 189v).

$\mathrm{Au}$ livre XVII, Barthélemi rapporte deux affirmations concernant la nature de l'herbe de coriandre: l'une prêtée à Galien, l'autre à Isidore; conformément à son habitude, Barthélemi ne tranche pas entre deux opinions pourtant radicalement opposées, la nature du coriandre étant dans un cas chaude, dans l'autre froide. Corbechon traduit le texte latin, mais rajoute sa propre impression, en tentant de réconcilier les deux autorités : « ...car il est froit, si qu'il tue les vers ou ventre, si comme dit Galien. Et si est si chaut qu'il esmeut les gens a luxure, si comme dit Ysidoire. Pour cecy accorder, je croy que herbe est froide et ce veut dire Galien, mais la semence est chaude, et de ce parloit Ysidoire » (A, fol. 284v). Toujours dans le livre sur les plantes, nous avons un cas frappant où Corbechon tranche avec assurance :

Nielle est en latin appellee gith, si comme dit le Plateaire et Aristote et Dyascorides ; et pour ce est elle cy mise entre les herbes dont les noms se commencent par .g. Nielle est une herbe chaude et seche ou second degré qui croist entre le blé et porte une petite graine noire qui a aussi comme la figure de un treangle et est un pou amere et a vertu de degaster les humeurs et de ouvrir les conduis de la rate et du foye, et de oster les vantosités et de guerir les emoroïdes, et de tuerles vers ou ventre, quant on la prend avec du miel. Et quant elle est cuite en vin aigre, elle tue les vers des oreilles quant on la gette tievde dedens. Aucuns dient que gith est une maniere de graine noire semblable au commin en quantité et le met on en pain pour 
adoulcir, si comme dit la glose sur le xxviii $^{\mathrm{e}}$. chappitre de Ysaïe le prophete, mais la premiere oppinion me plait mieulx (A, fol. 294v).

Il en va de même au livre XIX, dans la discussion de différents avis sur l'unité; Corbechon indique alors qu'il préfère celui de saint Bernard: «mais saint Bernart en son livre qu'il fist au pape Eugene si en parle plus proprement, et dist que il est une unité naturelle et une unité gracieuse...(A, fol. 393).

Les cas où Corbechon se fait critique d'assertions trouvées dans le De proprietatibus rerum sont assez rares. Le plus important est à propos des opinions politiques du traducteur de Charles V et de ses propos sur l'Angleterre ${ }^{16}$. La critique de Corbechon peut se faire indirecte; tel est le cas au livre VIII, lorsqu'il est question de la définition des différents cieux, qui pose en particulier le problème de la double nature du ciel, météorologique et théologique. Lorsqu'il est question du cinquième élément, Barthélemi emprunte au pseudo-Aristote, d'après le Liber de causis. Corbechon traduit, puis rajoute une sentence lapidaire finale qui clôt le débat, sur lequel Barthélemi laisse le lecteur: «...et pour ce dit Aristote en celuy livre que le Createur a mis le ciel commencement et cause de generacion et de corruption, par quoy il s'ensuit qu'il ne puet estre engendré ne corrompu, ou il fauldroit que la generacion et corruption des causes n'eüst point de fin, laquele chose est reprouvee par les sages philosophes » (A, fol.148v). Mais parfois, Corbechon s'exprime plus directement; tel est le cas au livre XVII, lorsqu'il manifeste clairement son opinion à propos de la "salverique »:

Salverique, si comme dit Plinius, est une petite herbe qui a moult de feuilles et de branches; et quant on la touche a la main, elle donne moult grant odeur et se tient a la terre a petites racines; et croist en lieux durs et plain de pierres et est une herbe chaude et seche et de substance bien soutille. La racine de ceste herbe cuite oste le vomir et si conforte moult l'estomac, si comme dit Plinius ou .vii ${ }^{\mathrm{e}}$. chappitre de son. $\mathrm{xx}^{\mathrm{e}}$. livre. Hugusse dist que salverique est une herbe poingnant qui en France est appellee chauchetreppe et est autrement appellee escorpion pour ce qu'elle point ceulx qui la touchent, ainsi comme fait l'escorpion. En cette matiere, je croy mieulx a l'oppinion de Plinius le naturien que a celle de Hugusse le grammarien (A, fol. 313v).

Que conclure de ce rapide parcours dans le Livre des proprietés des choses? Les exemples que nous venons de relever dans le précédent sondage montrent bien que, comme il avait été annoncé, Corbechon parcourt une palette assez large d'attitudes: il peut abréger par endroit, allonger à d'autres; préciser un propos confus de Barthélemi ou, au contraire, à la suite d'un résumé ou d'une omission, rendre obscur un passage latin difficile, mais initialement clair. De manière générale, on peut toutefois noter des tendances manifestes chez Corbechon. Il est méfiant envers tout ce qui peut être suspect aux yeux du dogme chrétien, réticent aux développements trop techniques ou trop abstraits. Il tente d'être vigilant et d'éviter les effets de la compilation cumulative, réorganisant parfois, résumant, regroupant. De manière générale, il apparaît clairement que Corbechon possède le désir de s'adresser à un lecteur qui n'est pas trop savant, mais qui désire acquérir au moins une coloration scientifique. Pour cela, le traducteur, chaque fois qu'il le peut, insère dans son propos quelque remarque, quelque exemple simple qui permette de souligner, de «faire parler» le discours encyclopédique. Il se complaît particulièrement à éviter le mot technique au profit d'un terme plus simple ou d'une périphrase explicative. Il remplit en cela la mission qui lui a été attribuée par le roi: en passant au vulgaire, l'encyclopédie s'«encyclopédise» 
davantage, au sens moderne. Elle conserve sa dimension de somme, tout en s'intégrant dans un tissu réceptif qui se fait plus large. En exagérant pour souligner, ou peut dire que la suite de la démarche de Corbechon serait l'adjonction de planches!

En ce qui concerne le genre encyclopédique, on peut me semble-t-il considérer l'entreprise de traduction de Jean Corbechon comme partie intégrante de la dynamique du genre. A une époque où celui-ci ne produit plus réellement, la traduction apporte une relance, c'est-à-dire surtout un horizon quelque peu nouveau. Cette voie ne sera pas véritablement suivie dans l'immédiat, et les dernières encyclopédies médiévales, de Pierre d'Ailly, de Polydore Virgile, de Gregor Reich, seront écrites en latin. De même qu'après Oresme, en dépit des avancées qu'il fit faire au vernaculaire, le latin continuera encore d'être le support langagier de la science. Mais, même si l'encyclopédisme vulgaire ne prend pas un réel essor, même si on lit davantage Barthélemi que Corbechon- d'après le témoignage quantitatif des manuscrits conservés -, il n'en demeure pas moins que le texte de Corbechon marque une étape dans l'histoire du genre. Même à l'état d'appendice, il revêt une importance considérable. Cette importance peut être mise dans la lumière des autres traductions vernaculaires du De proprietatibus rerum. Cet ensemble, réalisé dans le siècle et demi suivant l'écriture de l'original, apporte un témoignage important sur les mutations qui se sont opérées dans les milieux princiers, quant au désir de Lettres de ceux-ci. Il ne suffit plus de lire les textes courtois, en dépit même du renouveau qui, à travers les ordres, les "clubs", s'opère à la fin du Moyen Âge : l'Ordre de la Toison, de la Rose et tant d'autres marquent, en des périodes particulièrement troublées, le regard nostalgiques des puissants vers un état du monde évanoui. Mais, à côté de cette courtoisie réactionnaire, se glisse le texte à caractère savant, qui, dans son inéluctable présence, souligne bien que les enjeux ont changé. La turbulence militaire avait depuis longtemps fait feu, depuis Poitiers, et ce n'est pas Azincourt ou Nicopolis qui pouvaient en réactiver les cendres. C'est au niveau des rêveries de quelques princes fougueux, Armagnacs ou Bourguignons, au niveau d'une littérature seulement qu'elles pouvaient véritablement revivre. Si l'exploit militaire demeurait un possible enviable, le politique partout finissait par l'emporter. Le message de Charles $\mathrm{V}$ devait alors résonner dans la postérité.

L'évolution qui, creusée sous le règne du «roi sage », avait pris naissance dès la fin du XIII ${ }^{e}$ siècle, était inéluctable ; comme le disait un Latini, le prince devait connaître l'art suprême de la politique, art qui se bâtissait, selon l'ordo même du Tresor, sur la connaissance encyclopédique. Si le prince n'est pas bon latiniste, l'encyclopédie ira alors vers lui : tels sont les buts premiers du Livre dou Tresor, du Placides et Timéo, du Livre des proprietés des choses.

Le De proprietatibus rerum connaît ainsi, souvent imperceptiblement, une déviation, qui est marquée par le prologue de Corbechon. Ce dernier traduit assez fidèlement certes, mais surtout «clerement ». Par cet adverbe, ne doit-on pas comprendre justement qu'il faut rendre clair non seulement la connaissance en elle-même, mais aussi le message que le texte véhicule, message explicité à la dédicace? A la lecture de l'encyclopédie "translatée ", c'est-à-dire traduite, expliquée, simplifiée, agrémentée, parfois mise au goût du jour, le lecteur trouvera la summa brevis de ce qui lui sera nécessaire pour comprendre le monde. L'encyclopédie traduite conserve certes un des buts essentiels que la plupart des textes de ce genre se sont donnés: édifier, aider le lecteur à demeurer sur la voie de la vertu. Mais sans doute va-t-elle plus loin que celle du frère 
Mineur: car elle s'inscrit pleinement dans une dialectique savoir/pouvoir. Et sa diffusion, dont témoignent la beauté, la richesse des manuscrits, montre bien que c'est avant tout le prince et son entourage qui sont concernés: le prince qui doit être litteratus, l'entourage qui doit connaître pour gérer, conseiller.

Il est donc légitime de parler de dynamique, voire de re-création, à travers la traduction encyclopédique. En termes positivistes, le savoir n'évolue en rien, au contraire même: la contemporanéité qui s'intègre est, on l'a vu, au niveau de la remarque, de l'anecdote: pas d'impetus chez Corbechon, pas de théorie magnétique, pas de logique nominaliste; le traducteur n'entend pas les voix des Universités de Paris ou d'Oxford; s'il a probablement rencontré Nicole Oresme dans la proximité du roi, il n'en a pas écouté les leçons. Ce ne sont pas, historiquement, ses capacités personnelles qui sont en cause, c'est l'exercice auquel il se prête. Il est en cela au cœur du problème des traducteurs et au cœur de la démarche encyclopédique. Il doit traduire des termes latins, pour produire des termes français. Ceux-ci sont les composants d'une véritable «sur-transposition» qui s'opère à partir de la transposition encyclopédique latine hypotextuelle. Le moteur de cette translation/transposition, c'est la volonté exprimée par le roi, volonté à travers laquelle la nova encyclopedia doit remplir son rôle sur l'échiquier politique scripturaire. Et Corbechon s'acquitte correctement de sa tâche : à son insu, il fournit un nouveau produit qui, bien que calqué sur un texte, s'en éloigne de manière significative, car ce qui est en jeu en est une réception différente: pas de marginalia moralisés dans les manuscrits de Corbechon. Là, on cherche, directement, les informations sur le monde et sur la nature : accessoirement, le poète y trouvera une image; mais essentiellement, le conseiller, le notaire, l'écrivain faisant œuvre didactique et politique, le prince, le roi y trouveront l'assurance de l'autorité, les exemples nécessaires pour étayer leurs démonstrations, leurs négociations, leurs conseils ; et il s'agira encore là d'autorité, point de départ et point d'aboutissement de l'ensemble d'un genre qui part des écrits autorisés qu'il compile, pour fournir des arguments d'autorité, tout en ayant véhiculé aussi l'affirmation de l'autorité d'un «je » encyclopédiste.

\section{ANNEXES}

\section{DÉDICACE AU ROI CHARLES V DE JEAN CORBECHON}

(Ms. BN fr. 22531)

(fol.12) A trés hault et puissant prince Charles le Quint de son nom, par la divine pourveance de Dieu roy de France, paisible seignourie soit donnee de Celui par qui les roys regnent. Et de par le translateur de ce livre qui, pour cause de sa petitesce, nommer ne se doit, soit offerte et presentee honneur et reverence, subjection et obeissance en tous ses commandemens sanz contredit.

Selon la verité des divines Escriptures et humaines, entre toutes les humaines perfections que cuer royal doit desirer, le desir de sapience doit par raison tenir le 
premier lieu. La cause si est, car la noblesce de cuer royal doit souverainnement et premierement desirer a bien et honnourablement et justement regner et gouverner les subgez.

Et ce ne puet il faire sanz sapience par quoy il s'ensuit que, devant toutes choses aprés Dieu, il doit amer et desirer sapience. Et pourtant dit Salemon aux roys et aux princes, ou Livre de Sapience : « vous qui estes dessus les peuples et contenez les nacions, amez et desirez sapience et justice, a celle fin que vous et voz hoirs regnez perpetuelment ».

Ce desir avoient les nobles roys puissans qui, ou temps ancien, ont vaillanment gouverné le monde en divers lieux et en diverses regions; lesquelx ont dés leur enfance labouré par estude en diverses sciences pour acquerir la perfection de sapience, si comme dit Ptholomee, le roy d'Egipte qui fu trés grant philosophe et plus grant astrologien. Et fist venir en son royaume .lxx. bons clers des Juifs qui lui interpreterent et translaterent toute la Bible en son langage.

Et du grant roy Alixandre raconte Agelle le Philosophe que Aristote fu son maistre, et lui apprist philosophie et astrologie ; et disoit Phelippe son pere, le roy de Macedonie, qu'il avoit plus grant joye de ce que son filz estoit né ou temps d'Aristote que il ne avoit pour ce qu'il devoit estre son hoir et son successeur en son royaume. Et ce, disoit il pour la sapience qu'il pensoit que Aristote apprendroit a son filz Alixandre.

De Julius Cesar, empereur de Romme, avons nous en la premiere partie du Livre de la vie Cesar comment il estudia diligemment en diverses sciences et especialment en astrologie, et comment il trouva le cours du soleil et le bissexte en comptant les heures et les minutes. De ceste matiere et de pluseurs aultres il fist et escript pluseurs livres. Et de ce Julius Cesar parle Solinus en son premier livre, ou il dit que nul de lui ne lisoit plus prestement, nul n'escripvoit plus hastivement, nul ne dittoit plus proprement, et avenoit aucunefoiz que tout ensemble et a une foiz il dittoit quatre [fol.12v] epistres ou .iiii. lettres a quatre escripvains qui escripvoient de diverses matieres, desquelles l'une ne ressembloit l'autre.

De l'empereur Theodose lisons nous ou prologue de l'Istoire partie en .iii., que de jours il se excercitoit en armes, et de nuit il estudioit en livres. Et pour ce qu'il ne feüst ennuyé de son estude, il ne vouloit que ame demourast avec lui en son estude; maiz avoit un chandelier qui lui rendoit huile pour sa lumiere sanz administration d'autruy, et ainsi il ne chargoit nulluy, et si en estudioit plus paisiblement.

Du glorieux roy de France saint Charles lisons nous qu'il estudioit en pluseurs sciences, et avoit fait paindre en son palais trés richement les sept ars liberaulx a celle fin que quant il n'avoit loisir de les veoir en livres qu'il les veïst en painture. Il estudioit aussi moult voulentiers la doctrine saint Augustin, et par especial les livres de La cité de Dieu. Et pour l'amour qu'il avoit a sapience et pour l'onneur et prouffit du royaume de France, il fist transporter et translater l'estude de Romme a Paris. Et avoit un maistre que on appelloit Alquin, duquel il apprist logique, rectorique et astrologie ; en laquele astrologie il prouffita moult grandement et y fist moult de bonnes choses, selon ce que dit Vincent ou .xvii ${ }^{\mathrm{e}}$. livre de sa compilacion.

Et se la science d'astrologie fait a louer et a recommender en la personne du puissant roy de France Charles, je ne puis veoir que a nul de ses successeurs elle face a reprendre ne a blasmer, comme ainsi soit que pluseurs sainctes personnes (fol. 12vb) y aient estudié diligemment, si comme Abraham et Moyses qui furent moult expers en la 
sapience des Egiptiens, c'est a dire en astrologie, si comme nous lisons ou Livre des faiz des Apostres.

Or appert dont clerement que, entre les desirs humains de cuer royal, le desir de sapience doit estre le principal, si comme il estoit ou roy Salemon, qui a Dieu demanda qu'il lui donnast science et sapience par laquele il peüst gouverner son peuple justement; et Dieu lui donna un cuer sage et entendant, ainsi comme il est escript ou tiers Livre des Roys. Pour tant disoit Tulles, ou livre de ses Distinctions, que savoir estoit euvre royal. Et Seneque, ou livre de ses Epistres, si dit que le siecle estoit d'or quant les sages le gouvernoient.

Cest desir de sapience, prince trés debonnaire, a Dieu fichié, planté et enraciné en vostre cuer trés fermement dés vostre jeunesce, si comme il appert manifestement en la grant et copieuse multitude de livres de diverses sciences que vous avez assemblez et assemblez chascun jour par vostre fervent diligence, esquelz livres vous puisiez la parfonde eaue de sapience, au seau de vostre vif entendement, pour la espandre aux conseilz et aux jugemens au prouffit du peuple que Dieu vous a mis a gouverner.

Et pour ce que la vie d'un homme ne souffiroit mie pour lire les livres que vostre noble desir a assemblez, et par especial ou temps present vous ne les pouez veoir ne visiter pour cause de voz guerres, et de l'administracion de vostre royaume, et de pluseurs autres grandes et inevitables occupacions qui, chascun jour, sourdent et viennent a [fol 13] vostre grant magnificence, pour tant est venu en vostre noble cuer un desir d'avoir le Livre des proprietés des choses, lequel est aussi comme une somme general contenant toutes matieres. Car il traitte de Dieu et de Ses creatures, tant visibles comme invisibles, tant corporelles comme esperituelles, du ciel, de la terre et de la mer, de l'air et du feu et toutes les choses qui en eulz sont.

Et au desir que vostre royal cuer a de avoir ce livre puet on congnoistre evidemment, que vous estes habitué et revestu de l'abbit de sapience: car selon le philosophe Aristote, ou livre de la Metaphisique, il affiert au sage de savoir toutes choses; en ce dont que vous desirez ce livre qui traitte de toutes choses, vous monstrez que vous avez amour et plaisance a sapience.

Et pour vostre bon desir accomplir, il a pleü a vostre royal majesté a commander a moy, qui suis le plus petit de voz chappelains et vostre creature et la faitture de voz mains, que je translate le livre devant dit de latin en françoiz, le plus clerement que je pourray. Je dont, qui suis tenu de droit divin, humain et naturel de obeir a voz commandemens comme a mon droit seigneur naturel et comme a celui qui m'a fait tel comme je suis, reçoy liement et accepte ceste obedience, en suppliant humblement a vostre trés habondant pitié, qu'elle vueille et daigne prendre en gré le pouuoir de ma petitesse ; et se deffault y a, qu'il soit imputé a ma trés grant ignorance. Et se bien y a, qu'il soit attribué a vostre bon desir et a Celui de qui tout bien vient, lequel par Sa grace vous doint savoir, pouoir et vouloir de regner en ce monde paisiblement, et en l'autre monde avec lui sanz fin glorieusement.

\section{NOTES}

1. - Voir ce texte à la fin de l'article. 
2. - S. Lusignan, «La topique de la translatio studii et les traductions françaises de textes savants au XIV ${ }^{\mathrm{e}}$ siècle ", Traduction et traducteurs au Moyen Âge, éd. du CNRS, Paris, 1989, p. 307.

3. - Voir, pour ce qui concerne le livre XVII, G. Sodigné-Costes, « La botanique de Barthélemi l'Anglais mise en français par Jean Corbechon : traduction ou adaptation?», Colloque de Lille, Traduction, transcription, adaptation au Moyen Âge, 22-24 sept. 1994, Bien lire et bien aprandre.

4. - Pour alléger les notations, je désignerai par X le BN fr. 16993, par A le BN fr. 22531. Les extraits du latins seront faits à partir du BN lat. 16098.

5. - Corbechon explique en outre l'étymologie isidorienne qui ne rend rien en français.

6. - Notons qu'ici Corbechon tente de donner une explication liée au sens du mot arvum. 7. - Voir B. Ribémont, De natura rerum. Études sur les encyclopédies médiévales, Orléans, Paradigme, 1995, chap. X.

8. - Voici à titre d'exemple la fin du livre III : Hec autem dicta de anime et potentiarum ejus et proprietatibus et virtutibus et effectibus, quantum spectat ad hoc opusculum, jam sufficiant. Nunc autem de proprietatibus humani corporis, cujus anima actus est et perfectio, adjuvante Dei gratia aliqua sunt dicenda (fol. 17v).

9. - Exemple relevé par G. Sodigné-Costes ,art. cit.

10. - Voir G. Sodigné-Costes, ibid.

11. - Dans le Bn lat. 16098, la figure et le texte à partir de axis visualis sont dans la marge, de la main du scribe, du fol. 14.

12. - Voir l'édition de cette partie du Livre XIX d 'après BN lat. 16098 et BN fr. 22531 par S. Louis et M. Salvat dans PRIS-MA, 9, 1, janv.-juin 1993, pp. 79-100. Sur la qualité mathématique du De proprietatibus rerum, voir mon article, «Le nombre, la Bible et l'encyclopédiste (Barthélemi l'Anglais et la symbolique numérique) », Le Divin. Discours encyclopédiques, Cahiers Diderot nº 6, dir. D. Hüe, Orléans, Paradigme, 1994.

13. - A, fol. 38 et Cassiodore, De anima, 2, PL. 70, c. 1285.

14. - «...sicomme dit saint Jeroisme en la glose sur le .xlix ${ }^{\mathrm{e}}$. chapitre de Genesis, ou il met exemple de Ruben, le filz ainsné se Jacob, que pour cause de villennie qu'il feist a son pere en congnoissant charnelment une de ses concubines, il fu privé du royaume et de la dignité qui lui estoit deue et fu donné a Judas, son frere, par l'ordenance de Dieu et de Jacob leur pere. Les enfans, dont, qui par nature devroient estre nobles, riches et fians comme furent leurs peres, deviennent par leur coulpe povres chetifs et serfs d'aultry » (quaest in Gen., 49, 3-4 (PL. 113, c. 178), (A, fol. 106) ; "Derechief, il procure delices et richesces d'autruy labour, sicomme dit David ou Psaultier et sicomme il appert ou.$v^{\mathrm{e}}$. chapitre du livre de Daniel du roy Balthasar,qui buvoit et mengoit en vaisseaux d'or et d'argent,qu'il avoit prins ou temple de Dieu, quant il vit une main qui escripvoit en la paroy contre lui, que la fin de lui et de son royaume estoit venue "(Dan. 5.2-6,25-30) (A, fol. 109) ; «Derechief, il est convoiteux de gaaignier, car les biens de son seigneur, il convertist a son propre usage, sicomme nous lisons de Giry, le servant Helisee le prophete, qui soubz le nom de son seigneur, demanda robes et argent a Naaman le chevalier au roy de Surie et quant il les ot receu, il les muca et n'en dist oncques rien a Helisee, sicomme il est escript ou . $\mathrm{v}^{\mathrm{e}}$. chapitre du quart livre des Roys » (2Rois, 5.21-25) (A, fol. 106v).

15. - En. 4, 68sq, fol. 194v ; fol. 197 ; Georg. 1, 299, fol. 184v ; Georg. 2, 112 et 4, 124), (Buc., 5, 17) Puniceis quantum saliunca rosetis, fol. 195 (Georg. 2, 448) reproduit incomplètement 
par Barthélemi, fol. 197 Georg. 4, 169 ou bien En. 1, 436, reproduit par Barthélemi, fol. 198.

16. Voir M. Salvat, «Quelques échos des rivalités franco-anglaises dans les traductions du De proprietatibus rerum ", Bien dire et bien aprandre, 5, 1987, pp. 101-12.

\section{AUTEUR}

\section{BERNARD RIBÉMONT}

C.E.M.O. Université d'Orléans 